\title{
Cloud Analyst: An Insight of Service Broker Policy
}

\author{
Hetal V. Patel ${ }^{1}$, Ritesh Patel $^{2}$ \\ Student, U \& P U. Patel Department of Computer Engineering, CSPIT, CHARUSAT, Changa, Gujarat, India \\ Associate Professor, U \& P U. Patel Dept., of Computer Engineering, CSPIT, CHARUSAT, Changa, Gujarat, India
}

\begin{abstract}
Cloud computing is one of the most promising computing field, which has given the new vision to the computing field. Cloud computing has opened a door as a new model for hosting and delivering services over the Internet. The main aim of cloud computing is to provide the resources as a services to the client. The new concept of Federated Cloud Computing in which multiple datacenters are distributed over different regions. Since the evolution of Cloud Computing: load balancing, energy management, VM migration, brokerage policies, cost modelling and security issues are popular research topics in the field. Deployment of real cloud environment for testing or for commercial use is very costly. Cloud simulators help to model various cloud applications and it is very easy to analyse. In this survey, two cloud simulators: CloudSim and CloudAnalyst, with their overview are presented so it can be easily decided which one is suitable for particular research topic. And also the survey on the service broker policy, its issues and available solutions are presented. Because there is always been the requirement to select appropriate datacenter so that further tasks for processing the request should be carried out with efficiency in least response time. So the issue of selecting appropriate datacenter which is known as service broker policy is kind of important.
\end{abstract}

Keywords: Cloud Computing, Modeling and Simulation, Cloud Simulators, Datacenter, Service Broker Policy.

\section{INTRODUCTION}

Cloud computing has recently emerged as a new paradigm for hosting and delivering services over the Internet. Cloud computing is attractive to business owners as it eliminates the requirement for users to plan ahead for provisioning, and allows enterprises to start from the small and increase resources only when there is a rise in service demand [9].

"Cloud computing refers to computing on the Internet, as opposed to computing on a desktop." [1]

Nowadays applications are provided over the internet. So there rise the need for shifting computing resources to the service provider from the user's location. This concept known as "Cloud Computing". It fulfils client's requests on the basis of available resources. It provides great flexibility in terms of computing resources like storage, platform, software, power and bandwidth. Cloud provides better solutions to the clients of its services.

The main idea behind cloud computing is not a new one. John McCarthy in the 1960s already envisioned that computing facilities will be provided to the general public like a utility [2]. The term "cloud" has also been used in various contexts such as describing large ATM networks in the 1990s. However, it was after Google's CEO Eric Schmidt used the word to describe the business model of providing services across the Internet in 2006, that the term really started to gain popularity [9].

Cloud computing comes into focus only when you think about what IT always needs: a way to increase capacity or add capabilities on the fly without investing in new infrastructure, training new personnel, or licensing new software. Cloud computing encompasses any subscriptionbased or pay-per use service that, in real time over the Internet, extends IT's existing capabilities.

\section{RELATED WORK}

Cloud computing provides computing resources as a service over a network. As rapid application of this emerging technology in real world, it becomes more and more important how to evaluate the performance and security problems that cloud computing confronts.

Currently, modeling and simulation technology has become a useful and powerful tool in cloud computing research community to deal with these issues[11].

There have been many studies using simulation techniques to investigate behavior of large scale distributed systems, as well as tools to support such research. Some of these simulators are GridSim, MicroGrid, GangSim, SimGrid, CloudSim, CloudAnalyst, iCanCloud, NetworkCloudSim, GreenCloud, MDCsim, EMUSIM, GroundSim, MRCloudSim, DCSim, SimIC. While the first three focus on Grid computing systems, CloudSim is, for the best of our knowledge, the only simulation framework for studying Cloud computing systems [6].

CloudSim allows simulation of scenarios modelingIaaS, because it offers basic components such as Hosts, Virtual Machines that model the services [6].

In Section III, two cloud simulators and their main functionalities are discussed. In Section IV, the concept of service broker policy, its benefits, its issues and its simulation results are presented. In Section V, available solutions in terms of research paper are discussed. In Section VI, implementation details is discussed. And finally the conclusion of survey is presented. 


\section{Cloud Simulators}

\section{A. CloudSim:}

It is event driven simulator built upon GridSim. Its programming language is Java, because of OOP feature CloudSim modules can be easily extensible with user's requirement. It has some extra ordinary features: creating huge datacenters, unlimited number of VMs, federated policy, brokering policy. It supports the important feature of Cloud Computing pay-as-you-go model. [4]

In majority of research papers,CloudSim is used forVM Management. It consists three operations:

1. Host Overload/Underload Detection:It determines when host is considered as being overloaded or underloaded. It helps to decide that migration of one or more VMs from the overloaded host. In case of underloaded host, all VMs should be migrated and host should be switch to sleep mode.

2. VM Selection techniques: It helps to select the VM that should be migrated from an overloaded/underloaded host.

3. VM Placement techniques: It helps to find out new host for the VMs selected for migration from the overloaded and underloaded hosts.

So there are different techniques already available for VM Management. If someone want to focus on one of the activity or whole VM Management for his research then CloudSim provides best platform.

\section{B. CloudAnalyst:}

It is based upon CloudSim, adding some new features to 3 it. It is basically made for evaluating performance and cost of large scale geographically distributed cloud system that is having huge user workload based on different parameters. It has an attractive GUI facility and flexibility to configure any geographically distribute system such as setting hardware parameters i.e., storage, main memory, bandwidth etc.It gives the simulation results in terms of chart and table that includes cost, response time, datacenter processing time, and load over datacenter, etc. [4]

Mainly CloudAnalyst have two separate responsibility of VM management and service broker which is combinely available in CloudSim. So if someone wants to focus on particular one then he should go for CloudAnalyst. Because it provides easy access to add new service broker policy. It also having one extra feature that provides load balancing among VMs that can be consider as VM management in CloudAnalyst. [5]

In majority of research papers, CloudAnalyst is used for load balancing policy and service broker policy.

Load Balancing Policy: It helps to select VM for upcoming request from the user in the way that it balances the load among VMs.

Service Broker Policy (Datacenter Selection Policy): It helps to select datacenter. We will see this topic in brief because we have made survey for this topic.

\section{Service Broker Policy}

A service broker decides which datacenter should provide the service to the requests coming from each user. And thus, service broker controls the traffic routing between user and datacenters. So in simple words, it is datacenter selection policy.

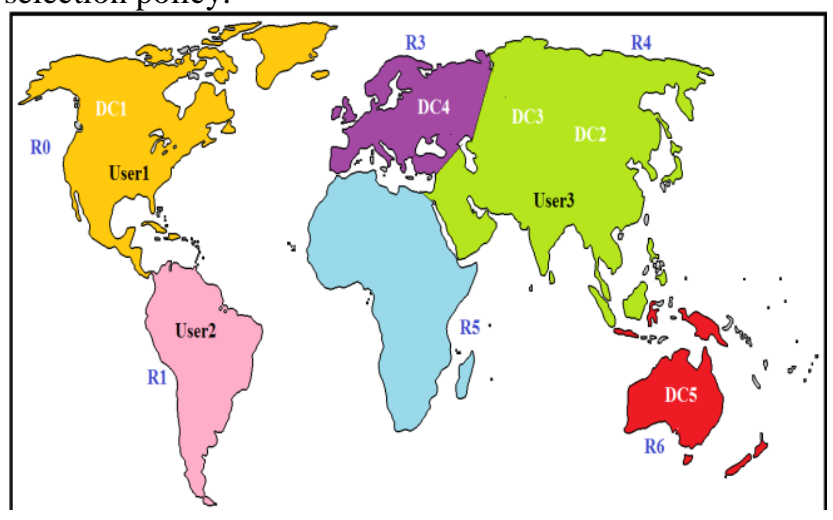

Fig. 1. Geographically distributed datacenters

From above figure, one can understand that in which kind of scenario service broker policy works.Here some datacenters and users are shown in the figure. When request come from the user then service broker policy helps to decide which datacenter will provide service for upcoming request.

There are three service broker policies already existing in CloudAnalyst [7]:

1. Closest Datacenter Policy

2. Optimize Response Time Policy

3. Dynamically reconfigurable routing with load balancing

A. Closest Datacenter Policy:

The datacenter which is having least proximity from the user is selected.Proximity in term of least network latency.If more than one Datacenters having same proximity then it will select datacenter randomly to balance the load.

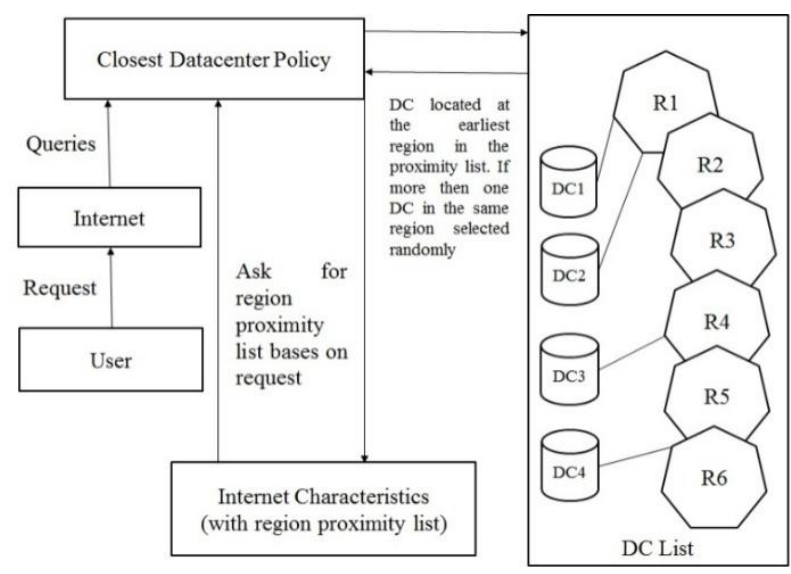

Fig. 2. Closest Datacenter Policy [7] 
B. Optimize Response Time Policy:

First it identifies the closest datacenter using previous policybut when Closest Datacenter's performance (considers response time) starts degrading it estimates current response time for each datacenter then searches for the datacenter which having least estimated response time. But there may be 50:50 chance for the selection of closest and fastest datacenter. (again here random selection)

C. Dynamically reconfigurable routing with load balancing:

This is an extension to Closest Datacenter Policy where the routing logic is similar.But it has one more responsibility of scaling the application deployment based on the load it is facing. It also increases or decreases the no. of VMs accordingly. This will be done taking under consideration the current processing times and best processing time ever achieved.This policy is under research so it gives useless results.

\section{Benefits of Service Broker Policy:}

1. By using Service Broker, traffic routing between user and datacenter is controlled.

2. It decides which datacenter should service the requests from each user. It means it provides flexible mapping of services to the available resources.

3. An efficient service broker policy ensures that the later tasks to proceed the request will be done efficiently and in least response time.

Practical comparison of these policies using CloudAnalyst:

Now in the below table the configuration is given which is used to take the simulation results for practical comparison of three service broker policy.

TABLE I

CONFIGURATION DETAILS

\begin{tabular}{|l|l|}
\hline Parameter & Value Used \\
\hline UB Name & UB1 \\
\hline Region & 2 \\
\hline Request Per User Per Hour & 60 \\
\hline Data Size Per Request & 100 \\
\hline Peak hour start(GMT) & 3 \\
\hline Peak hour end (GMT) & 9 \\
\hline Avg Peak Users & 40000 \\
\hline Avg Off Peak Users & 4000 \\
\hline DC 1 - No Of VM & 75 \\
\hline DC 2 - No Of VM & 50 \\
\hline DC 3 - No Of VM & 25 \\
\hline VM Image Size & $10000 \mathrm{MB}$ \\
\hline VM Memory & $512 \mathrm{MB}$ \\
\hline VM Bandwidth & $1000 \mathrm{bps}$ \\
\hline DC 1 - No Of Physical Machine & 2 \\
\hline DC 2 - No Of Physical Machine & 2 \\
\hline DC 3 - No Of Physical Machine & 2 \\
\hline DC - Memory Per Machine & $204800 \mathrm{Mb}$ \\
\hline DC - Storage Per Machine & $100000000 \mathrm{Mb}$ \\
\hline DC - Available BW Per Machine & 1000000 \\
\hline DC - No Of Processors Per Machine & 4 \\
\hline DC - Processor Speed & $10000 \mathrm{MIPS}$ \\
\hline DC - VM Policy & Time Shared \\
\hline User Grouping Factor & 1000 \\
\hline Request Grouping Factor & 100 \\
\hline Executable Instruction Length & 500 \\
\hline Load Balancing Policy & Throttled \\
\hline & \\
\hline
\end{tabular}

Copyright to IJARCCE

DOI 10.17148/IJARCCE.2015.4125
In CloudAnalyst, user base configuration and VM memory, image size, bandwidth should be define under Main Configuration tab. Datacenter configuration which consists no. of hosts, processor speed, memory, storage, bandwidth, VM policy should be define under Datacenter configuration tab. User grouping factor, request grouping factor, instruction length, load balancing policy should be define under Advanced tab.

Third policy gives useless results. So the comparison is done only between first two policies. The comparison is given below in terms of graphs for cost, response time and datacenter processing time.

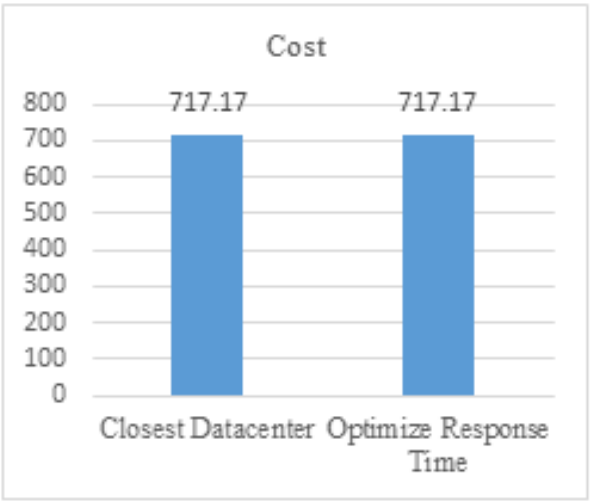

Fig. 3. Graph for cost (Comparison of policies)

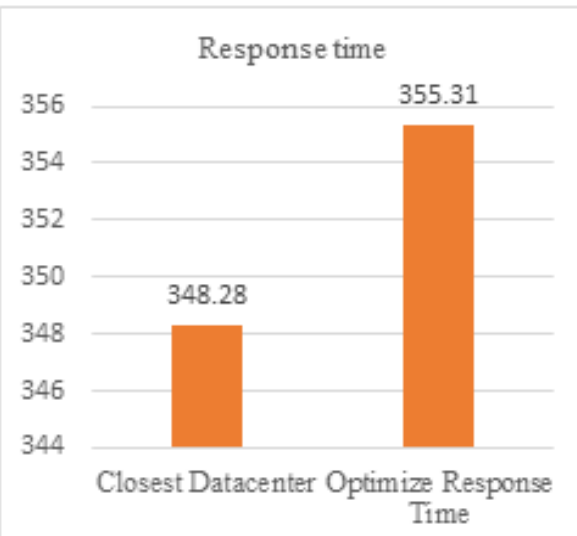

Fig. 4. Graph for Response Time (Comparison of policies)

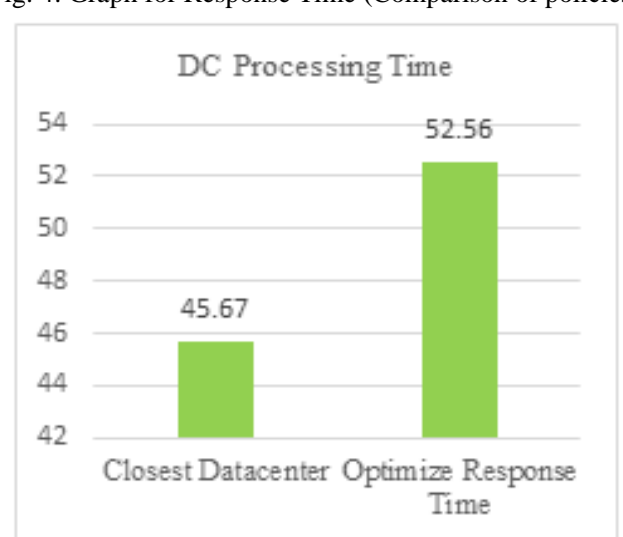

Fig. 5. Graph for DC Processing Time (Comparison of policies)

From above graphs we can say that closest datacenter gives the best results in terms of cost and response time compare to another two policies. 
Issues related to closest datacenter policy: [10]

Due to random selection of datacenter:

1. Possibility of selection of datacenter with higher cost.

2. For the same configuration, results may be differed.

3. There is also possibility of under-utilization of the resources.

\section{SOME Available Solutions}

There are some solutions available for solving the problems arises by random selection of datacenter. There are some solutions available in terms of research paper and we have implemented each of them except solution 4 in CloudAnalyst.

We have classified them into two categories: Static and Dynamic.

\section{A. Static Approaches: [7]}

Solution 1:

In this paper they have proposed that the datacenter having less cost will be selected.Here, only VM cost is considered. Using this solution, issue of random selection is solved, it becomes cost effective but data processing time is increased. For that they have proposed second algorithm. If the two most cost effective datacenters are selected then processing time is decreased but cost will be little higher than the previous one.

Simulation results:

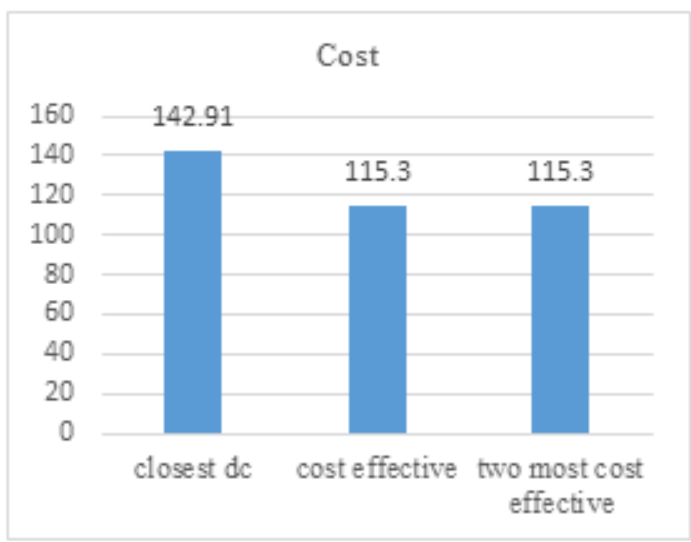

Fig. 6. Graph for Cost (Solution 1)

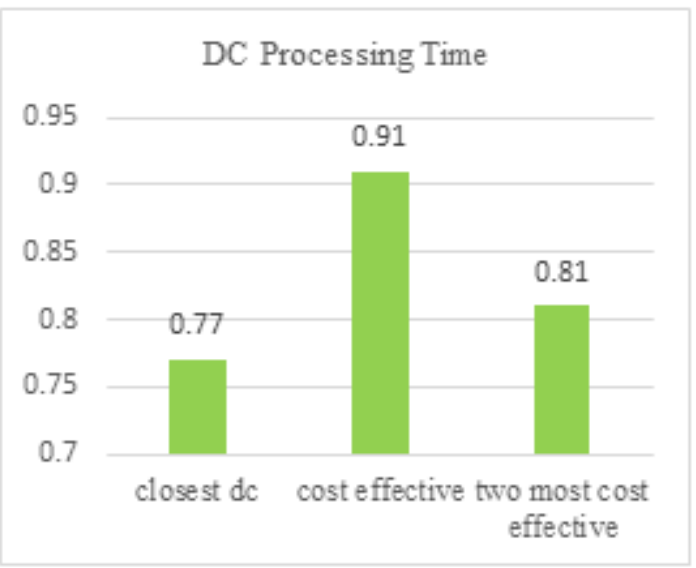

Fig. 7. Graph for DC Processing Time (Solution 1)
Solution 2: [10]

In this paper they have proposed that the datacenter selection will be done in Priority based Round Robin manner. Priority in terms of higher processing speed. It distributes requests uniformly among all the datacenters within a region.It leads to more resource utilization.But cost is increased.

Simulation results:

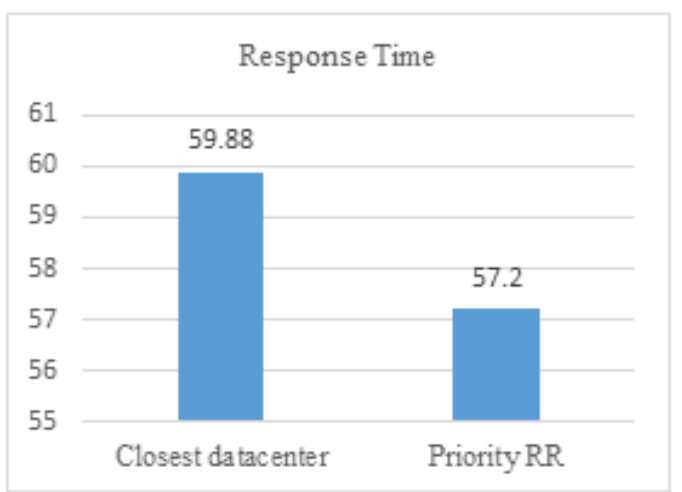

Fig. 8. Graph for Response Time (Solution 2)

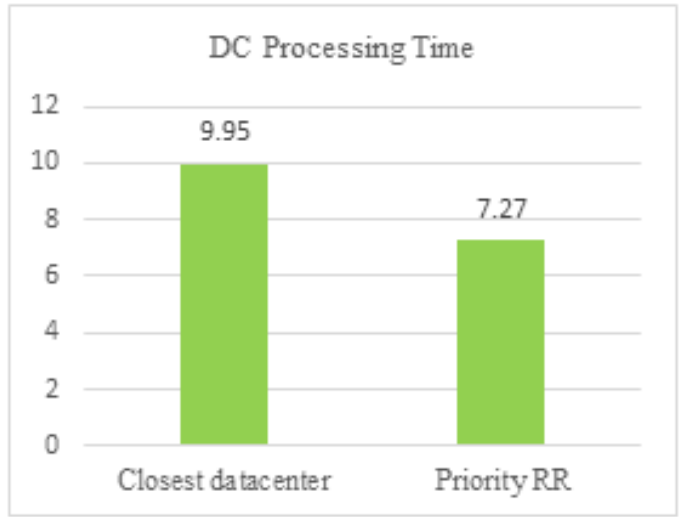

Fig. 9. Graph for DC Processing Time (Solution 2)

\section{B. Dynamic Approaches:}

Solution 3: [8]

Different datacenters may be of the same hardware configuration but contains virtual machines in varied number. So in this paper they have proposed that assign a proportion weight to the data centre according to no. of VMs it containing.According to that it handles the resources. It makes datacenter processing time less compare to existing ones.

Simulation results.

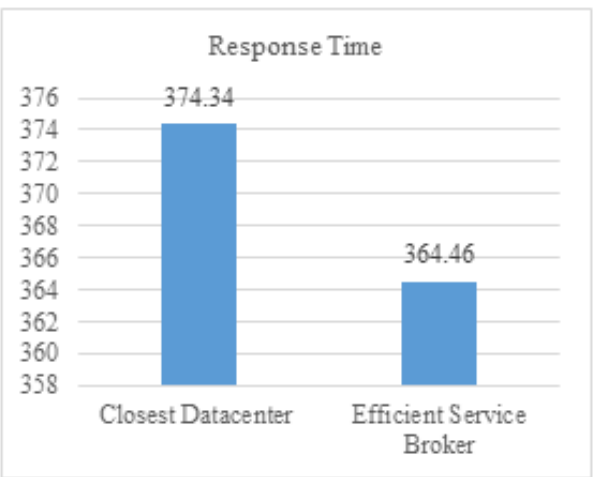

Fig. 10. Graph for Response Time (Solution 3 ) 


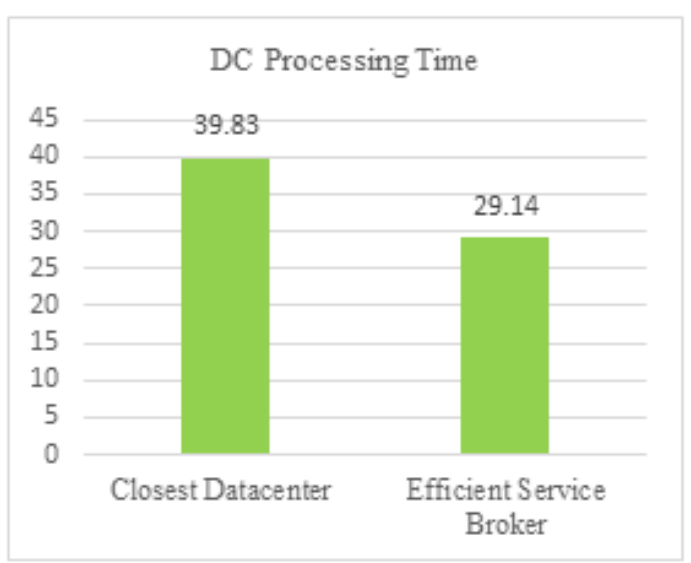

Fig. 11. Graph for DC Processing Time (Solution 3)

\section{Solution 4:[3]}

This algorithm selects datacenter based on two matrices values: Cost v/s Location and Performance v/s Availability. Here, cost means cost per VM, distance means network latency, performance means no. of jobs done per unit time, availability means no. of days available for launching new VMs within year. Find matrices values for each region and accordingly make list of datacenters for each matrix. Take intersection of this both list derived according to matrix value and then select datacenter from the intersected list. It gives good result for cost and performance both.

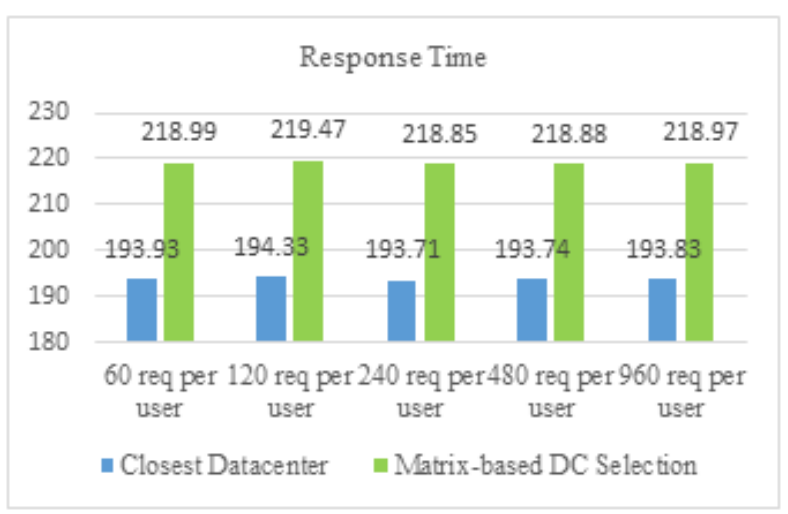

Fig. 12. Graph for Response time (Solution 4) [3]

TABLE II

Cost VAlues For DifFerent ReQuest Per USER VALues [3]

\begin{tabular}{|c|c|c|c|c|c|}
\hline $\begin{array}{c}\text { Request per } \\
\text { user }\end{array}$ & 60 & 120 & 240 & 480 & 960 \\
\hline $\begin{array}{c}\text { Closest } \\
\text { Datacenter }\end{array}$ & 42.52 & 49.09 & 62.27 & 88.45 & 140.95 \\
\hline $\begin{array}{c}\text { Matrix- } \\
\text { based DC } \\
\text { selection }\end{array}$ & 0 & 0 & 0 & 0 & 0 \\
\hline
\end{tabular}

\section{IMPlementation Of SERVICE Broker Policy IN Cloudanalyst}

For implementing new approaches for service broker policy, we have to make changes in following classes of CloudAnalyst:
TABLE III

Classes To Be Changed (For Gui)

\begin{tabular}{|c|c|c|}
\hline Package & Class & Method \\
\hline cloudsim.ext.gui.screens & $\begin{array}{c}\text { ConfigureSimulation } \\
\text { Panel }\end{array}$ & createMainTab \\
\hline cloudsim.ext & Constants & - \\
\hline cloudsim.ext & Simulation & runSimulation \\
\hline $\begin{array}{c}\text { cloudsim.ext.servicebro } \\
\text { ker }\end{array}$ & $\begin{array}{c}\text { Add new class } \\
\text { NewServiceBroker }\end{array}$ & - \\
\hline
\end{tabular}

TABLE IV

Classes To Be CHANGED (FOR NEW APPROACH)

\begin{tabular}{|c|c|c|}
\hline Package & Class & Method \\
\hline $\begin{array}{c}\text { cloudsim.ext.servicebrok } \\
\text { er }\end{array}$ & $\begin{array}{c}\text { NewServic } \\
\text { eBroker }\end{array}$ & getAnyDataCenter \\
\hline cloudsim.ext & Simulation & $\begin{array}{c}\text { createDatacenter } \\
\& \\
\text { runSimulation }\end{array}$ \\
\hline
\end{tabular}

\section{CONCLUSION}

From above discussion, we can conclude that CloudSim is limited to VM Management because in CloudSim brokerage policy is combinely given with VM Management which can't be easily modified. In CloudAnalyst these two facilities are separately given and it also provide geographically distributed cloud environment. So if we want to work particularly on service broker or load balancing then CloudAnalyst is best option. In both simulator there is no specific SLA parameter. But CloudSim is in developing mode and they are trying to include SLA parameter so in future it can be there in CloudSim.

From the survey of service broker policy we can conclude that we have to take care of two parameter cost and performance. Till now, whichever new service broker policy is proposed that improves either cost or performance. So when we try to propose new service broker policy we have to take care that both parameters can be improved.

\section{ACKNOWLEDGEMENT}

I would like to thank Dr.RajkumarBuyya, Professor of Computer Science and Software Engineering, and Director of the Cloud Computing and Distributed Systems (CLOUDS) Laboratory at the University of Melbourne, Australia; Rodrigo N. Calheiros research fellow at the Cloud Computing and Distributed Systems (CLOUDS) Laboratory at the University of Melbourne, Australia; Anton Beloglazov is a staff researcher at IBM Research.

I would like to thank Prof.Ritesh Patel, Associate Professor at U \& P U. Patel Department of Computer Engineering, CHARUSAT, Changa, Gujarat, India for all his diligence, guidance, and encouragement.

\section{REFERENCES}

[1] Cary Landis and Dan Blacharski: Cloud Computing Made Easy

[2] Parkhill D (1966): The challenge of the computer utility. AddisonWesley, Reading

[3] AmolJaikar, Seo-Young Noh. "Cost and performance effective data center selection system for scientific federated cloud", Springer Science+Business Media New York, Peer-to-Peer Netw. Appl. DOI 10.1007/s12083-014-0261-7, May 2014 
[4] Arif Ahmed, AbadhanSaumyaSabyasachi. "Cloud Computing Simulators: A Detailed Survey and Future Direction", IEEE International Advance Computing Conference, 2014

[5] B. Wickremasinghe, "CloudAnalyst: a cloudSim-based tool for modeling and analysis of large scale cloud computing environmens," MEDC Project Report, 2009.

[6] B. Wickremasinghe and R. N. Calheiros, "CloudAnalyst: a cloudSIm-based visual modeller for analysing cloud computing environments and applications," 24th International Conference on Advanced Information Networking and Application, pp.446-452, 2010.

[7] DhavalLimbani and BhaveshOza. A Proposed Service Broker Strategy in Cloudanalyst for Cost-effective Data Center Selection. In International Journal of Engineering Research and Applications, India, Vol. 2, Issue 1, pages 793-797, Feb 2012.

[8] KunalKishor, VivekThapar. "An Efficient Service Broker Policy for Cloud Computing Environment", International Journal of Computer Science Trends and Technology, Volume 2 Issue 4, pages 104-109,July-Aug 2014

[9] Qi Zhang, Lu Cheng, RaoufBoutaba. "Cloud computing: state-ofthe-art and research challenges", J Internet ServAppl (2010) 1: 7 18 , Springer

[10] Rakesh Kumar Mishra, Sandeep Kumar, SreenuNaik B (2014). "Priority Based Round-Robin Service Broker Algorithm for CloudAnalyst", IEEE International Advance Computing Conference, pages $878-881,2014$

[11] Wei Zhao, Yong Peng, Feng Xie, Zhonghua Dai (2012). "Modeling and Simulation of Cloud Computing: A Review", IEEE Asia Pacific Cloud Computing Congress, pages 20-24, 2012

\section{BIOGRAPHIES}

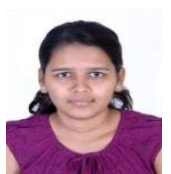

Miss Hetal Patel obtained her Bachelor's degree in Computer Engineering from DDU, Nadiad, Gujarat in 2009 and pursuing the Masters degree in Computer Engineering from CHARUSAT, Changa, Gujarat. Her research interest include Cloud Computing and Networking.

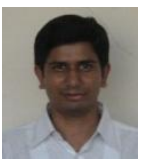

Mr.Ritesh Patel obtained his Bachelor's degree in Computer Engineering from Ganapat Univesity, Mehsana, Gujarat in 2002; Masters degree in Computer Engineering from DDU in 2004, Nadiad, Gujarat and pursuing PHD in area of Cloud Computing from CHARUSAT, Changa, Gujarat. Currently he is working as Associate Professor at U \& P U. Patel Department of Computer Engineering, CHARUSAT, Changa, Gujarat. His research interest include Parallel Computing, Next generation networks, Advanced Computer Architecture and Cloud Computing. 